06,12

\title{
Влияние наноконфайнмента на кинетику фазовых переходов в органическом сегнетоэлектрике DIPAI
}

\author{
() А.Ю. Милинский ${ }^{1}$, С.В. Барышников ${ }^{1}$, Е.В. Чарная ${ }^{2}$, И.В. Егорова ${ }^{1}$, Н.И. Ускова ${ }^{2,3}$ \\ ${ }^{1}$ Благовещенский государственный педагогический университет, \\ Благовещенск, Россия \\ ${ }^{2}$ Санкт-Петербургский государственный университет, \\ Санкт-Петербург, Россия \\ ${ }^{3}$ Санкт-Петербургский государственный университет аэрокосмического приборостроения, \\ Санкт-Петербург, Россия \\ E-mail: a.milinskiy@mail.ru
}

Поступила в Редакцию 19 фревраля 2020 г.

В окончательной редакции 19 февраля 2020 г.

Принята к публикации 20 февраля 2020 г.

\begin{abstract}
Представлены результаты исследования линейных и нелинейных диэлектрических свойств нового органического сегнетоэлектрика иодида диизопропиламмония (DIPAI), внедренного в пленки пористого оксида алюминия, в сравнении с объемным DIPAI. Для DIPAI в порах диаметром 300 и $60 \mathrm{~nm}$ обнаружено, что сегнетоэлектрическая фаза формируется в режиме нагрева и охлаждения в температурном интервале между двумя структурными фазовыми переходами выше комнатной температуры. Для обоих фазовых переходов не наблюдался заметный температурный гистерезис. Показано, что границы промежуточной полярной фазы для наноструктурированного DIPAI смещаются к низким температурам при уменьшении размера пор. Для объемного DIPAI выявлены два структурных перехода при нагреве с формированием промежуточной полярной фазы и только один переход при охлаждении, ниже которого возникало сегнетоэлектричество. Температура этого перехода была значительно меньше соответствующей температуры при нагреве. Предположено, что наблюдаемые различия фазовых переходов для DIPAI в порах и объемного DIPAI связаны с ускорением кинетики фазовых переходов в условиях наноконфайнмента.
\end{abstract}

Ключевые слова: органический сегнетоэлектрик, иодид диизопропиламмония, DIPAI, нанокомнфайнмент, кинетика фазовых переходов, диэлектрическая проницаемость, генерация третьей гармоники.

DOI: 10.21883/FTT.2020.07.49473.036

\section{1. Введение}

Сегнетоэлектрические материалы широко применяются в современных электронных устройствах. Хотя к настоящему времени открыто более сотни сегнетоэлектрических кристаллов, основное практическое применение получили неорганические сегнетоэлектрики со структурой перовскита, $\mathrm{LiNbO}_{3}, \mathrm{LiTaO}_{3}$ и их твердые растворы [1]. Органические сегнетоэлектрики меньше распространены из-за более слабой спонтанной поляризации, сравнительно низкой температуры Кюри и недостаточно сильного пьезоэлектрического эффекта. В то же время они обладают серьезными преимуществами, такими как малый вес и экологическая безопасность. Некоторые из этих веществ являются механически гибкими.

В последние годы было открыто несколько органических сегнетоэлектриков, принадлежащих семейству солей $\mathrm{C}_{6} \mathrm{H}_{16} \mathrm{~N} A$, где $A$ обозначает $\mathrm{Cl}, \mathrm{Br}$ и I [2-4]. Эти сегнетоэлектрики имеют достаточно высокие температуру Кюри и точку плавления. Наиболее высокую спонтанную поляризацию $P_{s} \sim 23 \mu \mathrm{C} \cdot \mathrm{cm}^{-2}$ демонстрирует бромид диизопропиламмония (DIPAB). Однако спонтанная поляризация в хлориде (DIPAC) и иодиде (DIPAI) диизопропиламмония также имеет величины, сравнимые с поляризацией такого известного сегнетоэлектрика, как $\mathrm{BaTiO}_{3}$, что делает их потенциальными материалами для применения в микро и наноэлектронике. В связи с перспективами практического использования новых органических сегнетоэлектриков, значительный интерес вызывают исследования влияния понижения размеров на их свойства. Фазовые переходы в наноструктурированных сегнетоэлектриках DIPAC и DIPAB изучались в [5-8]. Были обнаружены сдвиги температур и изменение последовательности сегнетоэлектрических фазовых переходов. Размерные эффекты в DIPAI, насколько нам известно, ранее не исследовались.

В настоящей работе приводятся результаты исследований линейных и нелинейных диэлектрических свойств DIPAI, внедренного в пленки пористого оксида алюминия с диаметром пор 300 и $60 \mathrm{~nm}$, в сравнении со свойствами объемного DIPAI.

\section{2. Образцы и методика эксперимента}

При комнатной температуре DIPAI принадлежит к орторомбической неполярной хиральной пространственной группе $P 2{ }_{1} 2_{1} 2_{1}[9,10]$. Однако при нагревании в 
зависимости от растворителя, в котором происходила перекристаллизация DIPAI, возможны два сценария. Согласно работе [9], в которой использовался этанол, увеличение температуры до $369 \mathrm{~K}$ приводит к возникновению полярной моноклинной фазы с симметрией $P 2_{1}$, стабильной до $415 \mathrm{~K}$. Выше $415 \mathrm{~K}$ DIPAI переходит В неполярную моноклинную фазу $P 2_{1} / \mathrm{m}$. В процессе охлаждения при $407 \mathrm{~K}$ наблюдается обратный переход из парафазы $P 21 / \mathrm{m}$ в сегнетоэлектрическую фазу $P 2_{1}$, в которой DIPAI остается вплоть до комнатной температуры.

При использовании раствора этанол-метанол $(1: 1)$ авторы работы [10] не обнаружили в полученных образцах полярной фазы $P 2_{1}$. При нагреве образца до температуры $376 \mathrm{~K}$ происходил фазовый переход из неполярной орторомбической фазы $P 22_{1} 22_{1}$ в неполярную моноклинную фазу $P 21 / \mathrm{m}$.

В наших исследованиях иодид диизопропиламмония был получен реакцией диизопропиламина с водным раствором иодоводородной кислоты (мольное соотношение $1: 1)$. В работе использовались диизопропиламин (SigmaAldrich, 99,95\%) и HI (Scharlab S.L., 57.0\%). Реакционную смесь выдерживали в течение $30 \mathrm{~min}$. Далее раствор фильтровался и твердый остаток перекристаллизовывался в горячем этиловом спирте при 350 К. При испарении образовывался бесцветный кристаллический порошок иодида диизопропиламмония, который сушился в эксикаторе над хлоридом кальция. Затем порошок промывался диэтиловым эфиром и снова сушился над хлоридом кальция. Согласно рентгеновскому спектру, полученный иодид диизопропиламмония при комнатной температуре находился в орторомбической фазе $P 2{ }_{1} 2_{1} 2_{1}$, что соответствует карточке CCDC 1518484.

В качестве объемных образцов DIPAI брались таблетки диаметром $10 \mathrm{~mm}$ и толщиной около $1.5 \mathrm{~mm}$, полученные путем прессования порошка при давлении $10000 \mathrm{~kg} / \mathrm{cm}^{2}$. Для получения нанокомпозитов использовались два вида пористых пленок $\mathrm{Al}_{2} \mathrm{O}_{3}$,TopMembranes Technology“ (Китай) толщиной $50 \mu \mathrm{m}$ с размером ячеек 450 и $125 \mathrm{~nm}$ и диаметром пор 300 и $60 \mathrm{~nm}$ соответственно (рис. 1). Внедрение сегнетоэлектрика в поры производилось из нагретого насыщенного раствора DIPAI в этаноле. Пленка пористого оксида алюминия помещалась в раствор, который медленно охлаждался и испарялся. Для удаления оставшегося этанола использовалась вакуумная сушка. После трехкратного повторения описанной процедуры степень заполнения пор, определенная по изменению массы пленок, составляла от 40 до $60 \%$.

Для измерения комплексной диэлектрической проницаемости применялся цифровой измеритель иммитанса E7-25 с частотным диапазоном $20-10^{6} \mathrm{~Hz}$ и рабочим напряжением $0.7 \mathrm{~V}$. Электродами служила серебряная паста. Температура определялась с помощью электронного термометра ТС-6621 с хромель-алюмелевой термопарой. Точность определения температуры составляла $0.1 \mathrm{~K}$. Исследования проводились в режиме „нагрев-

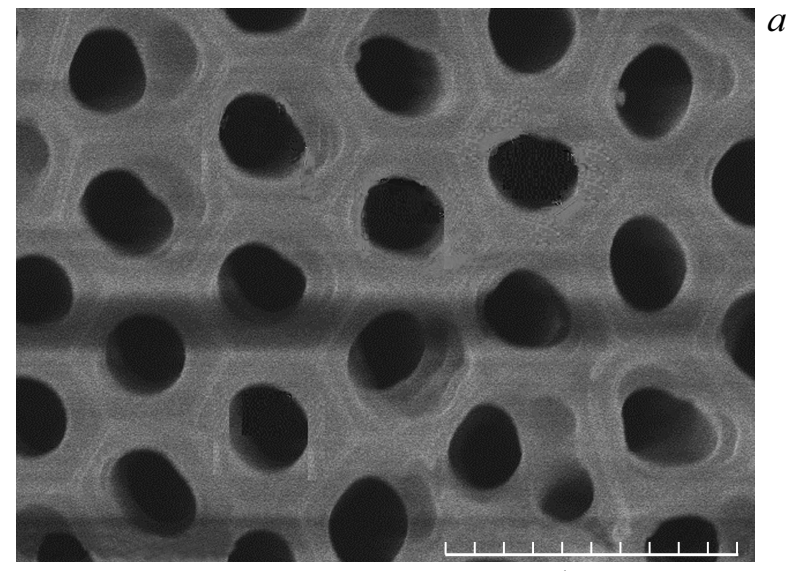

$1 \mu \mathrm{m}$

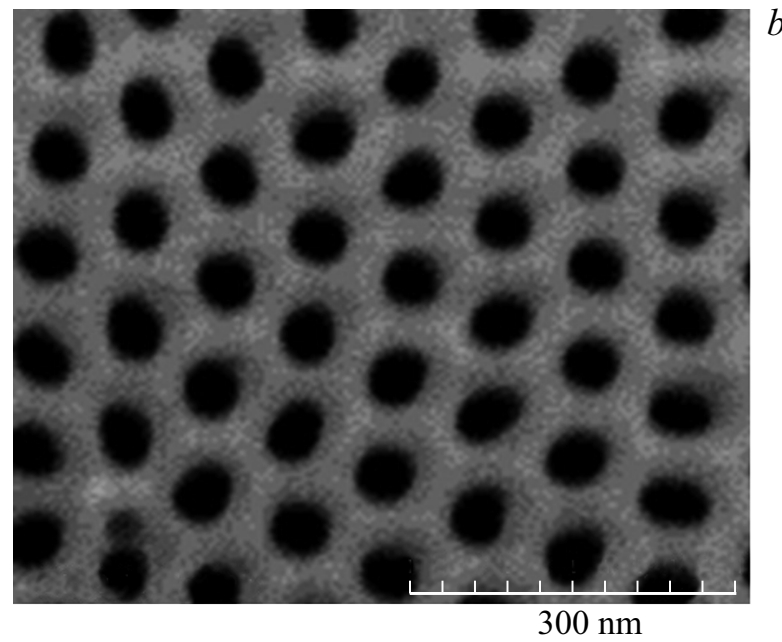

Рис. 1. Изображения поверхности пленок $\mathrm{Al}_{2} \mathrm{O}_{3}$, полученные с помощью электронного микроскопа: $a-$ размер пор $300 \mathrm{~nm}$, $b$ - размер пор $60 \mathrm{~nm}$.

охлаждение“ со скоростью $1 \mathrm{~K}$ в $\min$ в интервале от комнатной температуры до $440 \mathrm{~K}$.

Установка для исследований нелинейных диэлектрических свойств включала в себя генератор гармонических колебаний с рабочей частотой $2 \mathrm{kHz}$ [11]. Напряженность электрического поля при измерениях составляла около $300 \mathrm{~V} / \mathrm{cm}$. Сигнал снимался с резистора, включенного последовательно с образцом, и подавался на цифровой анализатор спектра, представляющий собой компьютер с 24-разрядным аналого-цифровым преобразователем ZET 230. Генерация третьей гармоники характеризовалась коэффициентом $\gamma_{3}=u_{3} / u_{0}$, где $u_{3}$ и $u_{0}$ - амплитуды третьей гармоники и сигнала на фундаментальной частоте.

\section{3. Экспериментальные результаты и обсуждение}

В сегнетоэлектриках смещение $D$ представляется в виде следующего ряда по степеням электрического 
поля $E[12]$ :

$$
\begin{aligned}
D & =P_{s}+\frac{\partial P}{\partial E} E+\frac{1}{2} \frac{\partial^{2} P}{\partial E^{2}} E^{2}+\frac{1}{6} \frac{\partial^{3} P}{\partial E^{3}} E^{3}+\ldots \\
& =P_{s}+\varepsilon_{1} E+\varepsilon_{2} E^{2}+\varepsilon^{3} E^{3}+\ldots,
\end{aligned}
$$

где $P$ - поляризация, $\varepsilon_{1}-$ линейная диэлектрическая проницаемость, $\varepsilon_{2}$ и $\varepsilon_{3}-$ диэлектрические проницаемости второго и третьего порядков, соответственно. В результате нелинейной зависимости $D$ от $E$ при приложении к образцу электрического поля $E=E_{0} \sin (\omega t)$ в токе через резистор будут присутствовать высшие гармоники на частотах $2 \omega, 3 \omega$ и т. д., амплитуды которых будут пропорциональны $\varepsilon_{2}, \varepsilon_{3}$ и т.д. соответственно. Как показано в работе [12], для сегнетоэлектриков с фазовым переходом первого или второго рода диэлектрическая проницаемость третьего порядка в рамках теории Ландау с точностью до слагаемых второго порядка по спонтанной поляризации дается выражением

$$
\varepsilon_{3}=\left[P_{s}^{2}\left(18 \chi \beta^{2}-10 \gamma\right) \beta\right] \chi^{4},
$$

где $\chi-$ восприимчивость, $\beta$ и $\gamma-$ коэффициенты в разложении Ландау при четвертой и шестой степени поляризации соответственно. Для переходов второго рода $\gamma=0$. Из выражения (2) следует, что генерация третьей гармоники значительно сильнее в сегнетоэлектрической фазе, в которой спонтанная поляризация не равна нулю, по сравнению с неполярной фазой. Поскольку восприимчивость имеет максимум около фазового перехода, то амплитуда третьей гармоники сначала нарастает в полярной фазе при удалении от фазового перехода, а затем уменьшается. Таким образом, исследование зависимости генерации третьей гармоники от температуры является удобным методом регистрации сегнетоэлектрического состояния.

Температурные зависимости реальной части диэлектрической проницаемости $\varepsilon^{\prime \prime}$ на частоте $10 \mathrm{kHz}$ и коэффициента третьей гармоники $\gamma_{3}$ для объемного DIPAI, полученные в режимах нагрева и последующего охлаждения после предварительного прогрева до $420 \mathrm{~K}$, показаны на рис. 2. При нагреве видны две аномалии на кривых диэлектрической проницаемости, обусловленные двумя структурными фазовыми переходами. Генерация третьей гармоники значительно возрастает в интервале между фазовыми переходами, что указывает на полярный характер промежуточной структурной модификации DIPAI. При охлаждении имеется только один переход, причем $\gamma_{3}$ растет ниже перехода. Нижний переход в сегнетоэлектрическое состояние в процессе нагрева происходит около $360 \mathrm{~K}$, следующий, из полярной фазы в неполярную, - около $380 \mathrm{~K}$. Сегнетоэлектрический фазовый переход при охлаждении виден около $360 \mathrm{~K}$, т.е. вблизи перехода в полярную фазу при нагреве. Выдержка образца при комнатной температуре более суток полностью переводит DIPAI в неполярную фазу,
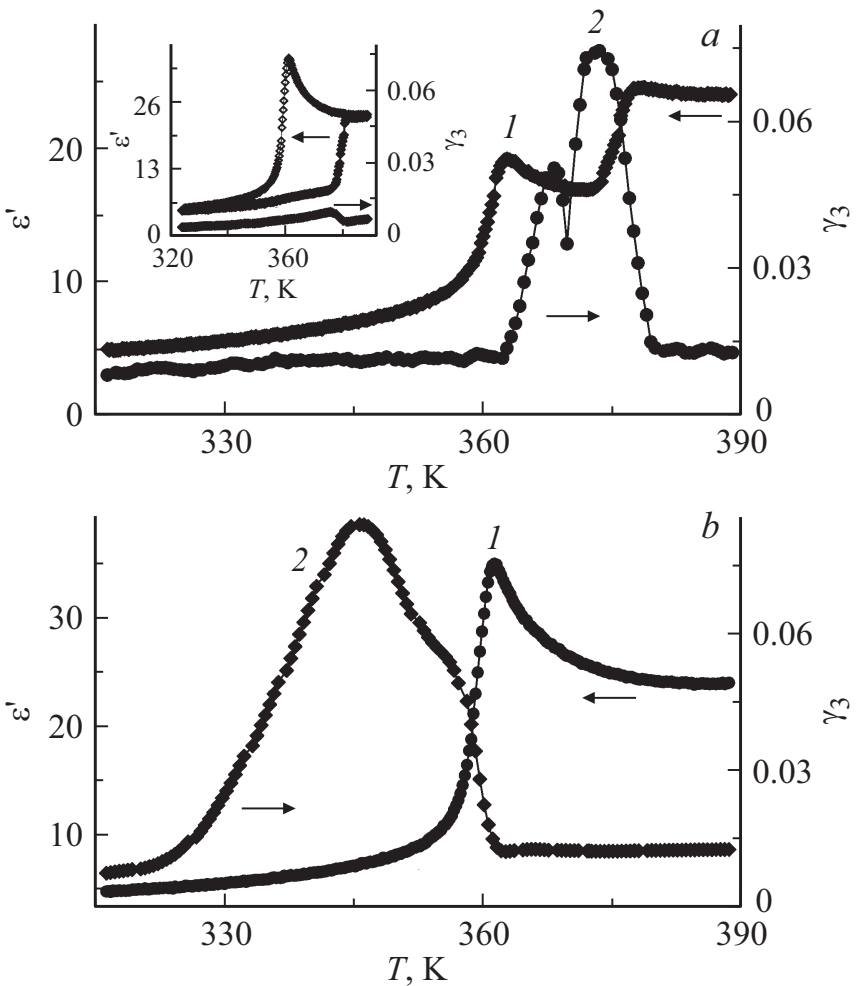

Рис. 2. Температурные зависимости вещественной части диэлектрической проницаемости $\varepsilon^{\prime}$ на частоте $10 \mathrm{kHz}(1)$ и коэффициента генерации третьей гармоники $\gamma_{3}(2)$, измеренные при нагреве $(a)$ и охлаждении $(b)$ для объемного DIPAI после прогрева до $420 \mathrm{~K}$. На вставке показаны зависимости от температуры $\gamma_{3}$ при нагреве (нижняя кривая) и $\varepsilon^{\prime}$ (верхние кривые) при нагреве и охлаждении (закрытые и открытые символы соответственно), полученные без предварительного прогрева.

и коэффициент $\gamma_{3}$ не растет при нагреве вплоть до фазового перехода около $360 \mathrm{~K}$. Для сравнения на вставке к рис. 2, $a$ приведен график зависимости диэлектрической проницаемости от температуры для этого образца непосредственно после синтеза без предварительного прогрева до $420 \mathrm{~K}$. В таком случае наблюдается только один структурный переход как при нагреве, так и при охлаждении. Коэффициент третьей гармоники невелик и слабо зависит от температуры, что означает отсутствие сегнетоэлектричества и выше, и ниже фазового перехода. В соответствии с литературными данными [9], переходы в предварительно прогретом образце соответствуют изменению структуры $P 2_{1} 2_{1} 2_{1} \rightarrow P 2_{1} \rightarrow P 2_{1} / \mathrm{m}$ (нагрев) и $P 2_{1} / \mathrm{m} \rightarrow P 2_{1}$ (охлаждение), а в непрогретом образце $P 2_{1} 2_{1} 2_{1} \rightarrow P 2_{1} / \mathrm{m}$ (нагрев) и $P 2_{1} / \mathrm{m} \rightarrow P 2_{1} 2_{1} 2_{1}$ (охлаждение).

Результаты исследования температурных зависимостей вещественной части диэлектрической проницаемости и коэффициента третьей гармоники для нанокомпозитов DIPAI $/ \mathrm{Al}_{2} \mathrm{O}_{3}$ представлены на рис. 3. Для заполненных пленок с обоими размерами пор (300 и $60 \mathrm{~nm}$ ) как при нагреве, так и при охлаждении 

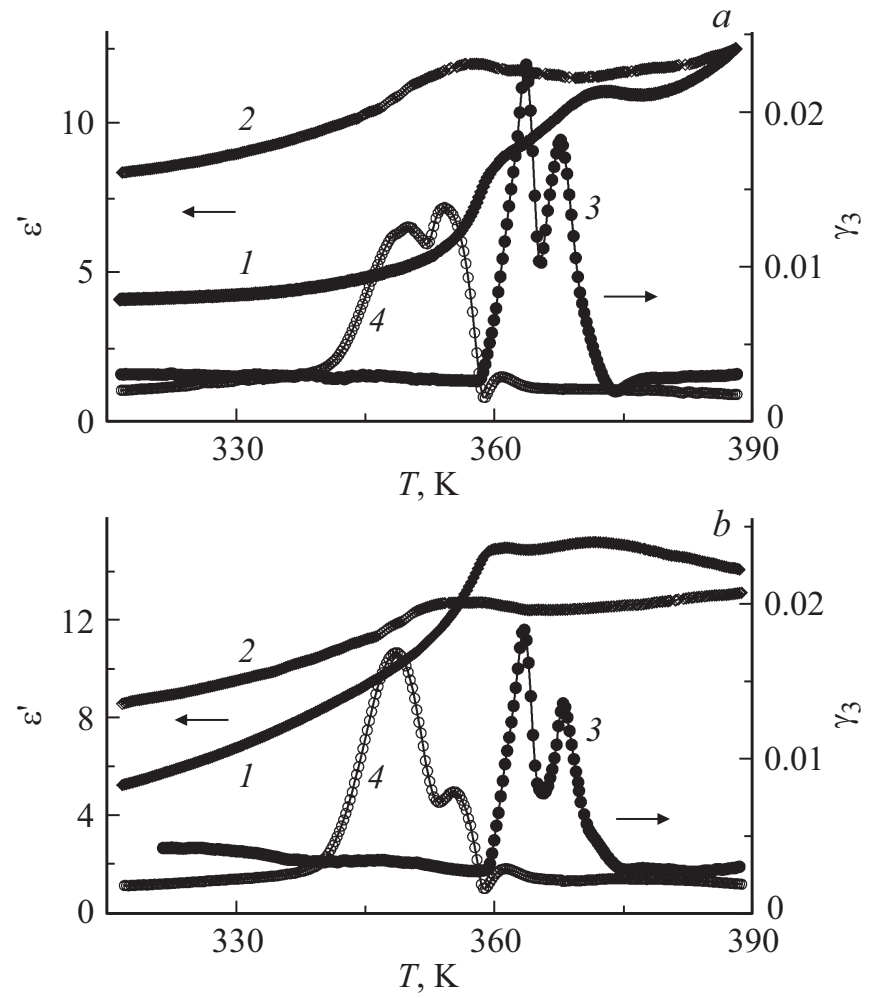

Рис. 3. Температурные зависимости вещественной части диэлектрической проницаемости $\varepsilon^{\prime}$ на частоте $10 \mathrm{kHz}(1,2)$ и коэффициента генерации третьей гармоники $\gamma_{3}(3,4)$, измеренные при нагреве $(a)$ и охлаждении $(b)$ для DIPAI в порах $300 \mathrm{~nm}(1,3)$ и $60 \mathrm{~nm}(2,4)$.

наблюдаются два фазовых перехода независимо от предварительного прогрева образцов. Особенности на кривых диэлектрической проницаемости существенно размываются в сравнении с объемным DIPAI. Если для DIPAI в порах диаметром $300 \mathrm{~nm}$ диэлектрическая проницаемость демонстрирует два отчетливых фазовых перехода, то уже для DIPAI в порах $60 \mathrm{~nm}$ идентифицировать наличие фазовых переходов по зависимости диэлектрической проницаемости от температуры практически невозможно. В этом случае именно измерения коэффициента третьей гармоники дают достоверную информацию о температурах и количестве переходов. Рис. 3 показывает, что сегнетоэлектрическая фаза возникает в наноструктурированном DIPAI при нагреве и при охлаждении. Видимый температурный гистерезис отсутствует. По аналогии с данными для объемного DIPAI следует предположить последовательность изменения структуры DIPAI в порах вида $P 2{ }_{1} 2_{1} 2_{1} \leftrightarrow P 2_{1} \leftrightarrow P 2_{1} / \mathrm{m}$.

Два структурных перехода для DIPAI в пористом оксиде алюминия сдвигаются к низким температурам по сравнению с фазовыми переходами, наблюдаемыми при нагреве в объемном, предварительно прогретом, DIPAI. Сдвиг растет с уменьшением размера пор. При этом температурная область существования се- гнетоэлектрической фазы практически не изменяется и остается равной примерно 20 градусам. Смещения температур структурных фазовых переходов могут быть обусловлены размерными эффектами. В этом случае их трактовка часто основывается на теории Ландау или модели Изинга [13,14]. Кроме этого, на температуру сегнетоэлектрического фазового перехода в условиях наноконфайнмента может оказывать влияние геометрия пор, взаимодействие наночастиц в порах между собой, механические напряжения, а также поляризация стенок пор [15-17]. Однако отсутствие видимого изменения температурного диапазона существования сегнетоэлектричества в наноструктурированном DIPAI для разного размера пор, по- видимому, требует специального рассмотрения.

Обращает на себя различие структурных изменений в объемном и наноструктурированном DIPAI. Для DIPAI в пористом оксиде алюминия отсутствует заметный температурный гистерезис фазовых переходов. Как при нагреве, так и при охлаждении переходы происходят при практически одинаковых температурах с формированием промежуточной полярной моноклинной фазы $P 2_{1}$. Картина переходов близка к той, которая наблюдается в объемном предварительно прогретом DIPAI при нагреве. Отсутствие гистерезисных явлений для DIPAI в условиях наноконфайнмента является характерным для фазовых переходов первого рода, близкого ко второму, и переходов второго рода с обычной быстрой кинетикой. В противном случае замедленной кинетики фазовых переходов появляется температурный гистерезис, величина которого зависит от скорости изменения температуры. Если имеется последовательность фазовых переходов, то границы температурных гистерезисов могут определяться областями существования определенных структурных модификаций. Такая ситуация наблюдалась, например, в кристаллах $\mathrm{LiKSO}_{4}$ [18]. Результаты, показанные на рис. $2, b$, для объемного DIPAI указывают на медленную кинетику переходов в режиме охлаждения. При охлаждении неполярная моноклинная фаза $P 2_{1} / \mathrm{m}$ сохраняется в переохлажденном состоянии вплоть до температуры, соответствующей равновесному переходу из сегнетоэлектрической моноклинной фазы в неполярную ромбическую фазу. Это стимулирует фазовую перестройку из переохлажденной фазы $P 2_{1} / \mathrm{m}$ в полярную фазу $P 2_{1}$. Фаза $P 2_{1}$ ниже $360 \mathrm{~K}$ находится в переохлажденном состоянии. При приближении к комнатной температуре в процессе дальнейшего охлаждения объемный DIPAI постепенно переходит в неполярную ромбическую фазу, о чем свидетельствует уменьшение коэффициента генерации третьей гармоники до значений, наблюдавшихся при нагреве. Подтверждением медленной кинетики фазовых переходов в объемном DIPAI при охлаждении служат также проведенные нами дополнительные измерения со скоростью изменения температуры $0.2 \mathrm{~K} / \mathrm{min}$ (вместо $1 \mathrm{~K} / \mathrm{min}$ ). В этом случае температура перехода в полярную фазу при охлаждении возросла на 5 градусов, а уменьшение коэффициента 
генерации третьей гармоники до значений, измеренный при нагреве, произошло на 10 градусов выше, чем в режиме более быстрого изменения температуры.

Причины, по которым в непрогретом предварительно объемном DIPAI не формируется сегнетоэлектрическая фаза, требуют отдельного изучения и анализ этого вопроса выходит за рамки настоящей статьи.

\section{4. Заключение}

Таким образом, перестройки структуры в объемном DIPAI и DIPAI в пористом оксиде алюминия существенно различаются. Для объемного DIPAI сегнетоэлектрическая фаза формируется после предварительного прогрева до $420 \mathrm{~K}$. При нагреве наблюдаются два структурных фазовых перехода, между которыми существует полярная фаза. При охлаждении виден только один переход, ниже которого существует сегнетоэлектричество. Температуры переходов между высокотемпературной неполярной моноклинной фазой и сегнетоэлектрической фазой различаются при нагреве и охлаждении примерно на 20 градусов. Для DIPAI в пористом оксиде алюминия сегнетоэлектричество наблюдается между двумя структурными переходами как при нагреве, так и при охлаждении. Область существования сегнетоэлектричества смещается к низким температурам при уменьшении размера пор. Для переходов отсутствует заметный температурный гистерезис. Полученные результаты демонстрируют медленную кинетику переходов для объемного образца и значительное ускорение кинетики переходов для наночастиц в порах.

\section{Финансирование}

Работа выполнена при поддержке Российского фонда фундаментальных исследований, грант № 19-29-03004.

\section{Конфликт интересов}

Авторы заявляют, что у них нет конфликта интересов.

\section{Список литературы}

[1] Physics of ferroelectrics. A modern perspective / Ed. K.M. Rabe, C.H. Ahn, J.-M. Triscone. Springer, Berlin (2007) $388 \mathrm{p}$.

[2] D.-W. Fu, W. Zhang, H.-L. Cai, J.-Z. Ge, Y. Zhang, R.-G. Xiong. Adv. Mater. 23, 5658 (2011).

[3] D.-W. Fu, H.-L. Cai, Y. Liu, Q. Ye, W. Zhang, Y. Zhang, X.-Y. Chen, G. Giovannetti, M. Capone, J. Li, R.-G. Xiong. Science 339, 425 (2013).

[4] A. Piecha, A. Ga̧gor, R. Jakubas, P. Szklarz. Cryst. Eng. Commun. 15, 940 (2013).

[5] S.V. Baryshnikov, E.V. Charnaya, A.Yu. Milinskiy, V.A. Parfenov, I.V. Egorova. Phase Trans. 91, 293 (2018).

[6] S.V. Baryshnikov, A.Y. Milinskii, E.V. Charnaya, I.V. Egorova. Phys. Solid State 61, 134 (2019).
[7] A.Y. Milinskii, S.V. Baryshnikov, E.V. Charnaya, I.V. Egorova, H.T. Nguyen. J. Phys: Condens. Matter 31, 485704 (2019).

[8] N.I. Uskova, E.V. Charnaya, D.Yu. Podorozhkin, S.V. Baryshnikov, A.Yu. Milinskiy, I.V. Egorova, D.Yu. Nefedov. Appl. Magn. Reson. DOI: 10.1007/s00723-019-01168-y.

[9] R.K. Saripalli, S. Diptikanta, S. Prasad, H. Nhalil, H.L. Bhat, T.N. Guru Row, E. Suja. J. Appl. Phys. 121, 114101 (2017).

[10] A. Piecha-Bisiorek, A. Ga̧gor, D. Isakov, P. Zieliński, M. Gałązka, R. Jakuba. Inorg. Chem. Front. 4, 553 (2017).

[11] A.Yu. Milinskii, S.V. Baryshnikov, V.A. Parfenov, S.A. Kozlola, N.H. Thuong. Tran. Elect. Electron. Mater. 19, 201 (2018).

[12] S. Ikeda, H. Kominami, K. Koyama, Y.J. Wada. Appl. Phys. 62, 3339 (1987).

[13] W.L. Zhong, Y.G. Wang, P.L. Zhang, B.D. Qu. Phys. Rev. B 50, 698 (1994).

[14] C.L. Wang, Y. Xin, X.S. Wang, W.L. Zhong. Phys. Rev. B 62 , 11423 (2000).

[15] A.V. Uskov, E.V. Charnaya, A.L. Pirozerskii, A.S. Bugaev. Ferroelectrics 482, 70 (2015).

[16] D. Yadlovker, S. Berger. Phys. Rev. B 71, 184112 (2005).

[17] V. Fridkin, S. Ducharme. Ferroelectricity at the nanoscale. Basics and applications. Springer-Verlag: Berlin, Heidelberg (2014). 122 p.

[18] B.F. Borisov, E.V. Charnaya, A.K. Radzhabov. Phys. Status Solidi B 181, 337 (1994).

Редактор К.В. Емцев 